PROCEEDINGS OF THE

AMERICAN MATHEMATICAL SOCIETY

Volume 126, Number 7, July 1998, Pages 1897-1900

S 0002-9939(98)04302-0

\title{
ON AN ISOMORPHISM PROBLEM FOR ENDOMORPHISM NEAR-RINGS
}

\author{
GARY L. PETERSON
}

(Communicated by Lance W. Small)

\begin{abstract}
Suppose $R$ and $S$ are endomorphism near-rings generated by groups of automorphisms containing the inner automorphisms of two respective finite perfect groups $G$ and $H$. In this note we show that if $R$ and $S$ are isomorphic, then $G / Z(G)$ and $H / Z(H)$ are isomorphic.
\end{abstract}

\section{INTRODUCTION}

In its most general form, the type of isomorphism problem referred to in the title is the following: Given two isomorphic near-rings $R$ and $S$ and two faithful modules $G$ and $H$ of $R$ and $S$, respectively, are there canonical normal subgroups $N$ of $G$ and $M$ of $H$ so that $G / N$ and $H / M$ are isomorphic? To give a specific illustration, in [1] Lyons and Mason asked: If the endomorphism near-rings $I(G)$ and $I(H)$ generated by the inner automorphisms of two finite groups $G$ and $H$, respectively, are isomorphic, is it true that $G / Z(G)(Z(G)$ the center of $G$ ) and $H / Z(H)$ are isomorphic? In [5], S. A. Syskin showed that this is the case if $G$ is strictly nonabelian (meaning that every normal subgroup of $G$ coincides with its commutator subgroup, or equivalently, that $G$ has no abelian chief factors). However, the answer to the Lyons-Mason question in general is no. Indeed an example is given in [5] of two nonisomorphic centerless solvable finite groups $G$ and $H$ with $I(G)$ isomorphic to $I(H)$. It must also be pointed out (and this is noted in [5]) that a stronger version of Syskin's strictly nonabelian result appears in [7] where it is shown that any two faithful modules of a 2-tame ring-free near-ring $N$ are finite and $N$-isomorphic when $N / J_{2}(N)$ has the descending chain condition on right ideals.

The purpose of this note is to prove the following theorem giving us another generalization of Syskin's strictly nonabelian result. In the statement of this theorem, an automorphism near-ring refers to an endomorphism near-ring of a group $G$ that is generated by automorphisms of $G$. Also, we shall normally use the notation and terminology of [3] (in particular, our groups are additive but not necessarily abelian) with the exception of following Scott's convention [6] of calling an endomorphism near-ring of a group $G$ containing the inner automorphisms of $G$ compatible instead of tame as is done in [3]. Finally, recall that a perfect group is one that coincides with its commutator subgroup.

Received by the editors June 30, 1996 and, in revised form, December 6, 1996.

1991 Mathematics Subject Classification. Primary 16Y30; Secondary 20 E36.

Key words and phrases. Near-ring, endomorphism near-ring.

(C)1998 American Mathematical Society 
Theorem 1. Suppose that $G$ and $H$ are perfect finite groups and that $R$ and $S$ are compatible automorphism near-rings of $G$ and $H$, respectively. If $R$ and $S$ are isomorphic, then $G / Z(G)$ and $H / Z(H)$ are isomorphic.

Of course, notice as immediate corollary we have an affirmative answer to the Lyons-Mason question for finite perfect groups:

Corollary 2. If $G$ and $H$ are finite perfect groups with $I(G)$ isomorphic to $I(H)$, then $G / Z(G)$ is isomorphic to $H / Z(H)$.

\section{The Proof of TheOrem 1}

We begin with two lemmas about monogenic modules.

Lemma 3. Suppose that $G$ is a monogenic $R$-module of a near-ring $R$. Then every distributive element of $R$ acts as an endomorphism of $G$.

Proof. Suppose that $\alpha$ is a distributive element of $R$ and that $G=g R$ where $g \in G$. If $x, y \in G$, writing $x=g \beta$ and $y=g \gamma$ where $\beta, \gamma \in R$ gives us

$$
(x+y) \alpha=(g \beta+g \gamma) \alpha=g(\beta+\gamma) \alpha=g(\beta \alpha+\gamma \alpha)=g \beta \alpha+g \gamma \alpha=x \alpha+y \alpha
$$

and hence $\alpha$ acts as an endomorphism of $G$.

Lemma 4. Suppose that $R$ is a compatible endomorphism near-ring of a group $G$ and suppose that $R$ has dccr. Then $G$ is a monogenic $R$-module if and only if $G / G^{\prime}$ is a monogenic $R$-module.

Proof. The fact that $G / G^{\prime}$ is monogenic when $G$ is monogenic is trivial, so assume that $G / G^{\prime}$ is monogenic. Let $N$ be the intersection of the maximal $R$-ideals of $G$. Since $(G / M) J_{2}(R)=0$ for any maximal $R$-ideal $M$ of $G,(G / N) J_{2}(R)=0$. From [2, Lemma 3.9], $G / N$ is its own socle and hence we can write

$$
G / N=\oplus \sum_{i \in I} N_{i} / N
$$

where $\left\{N_{i} \mid i \in I\right\}$ is a set of $R$-ideals of $G$ with each $N_{i} / N$ a minimal (and hence monogenic) $R$-module. Since $G / G^{\prime}$ is monogenic, it follows that one $N_{i} / N$, say $N_{1} / N$, is abelian and the rest of these factors are nonabelian.

We next note that $N_{i} / N$ is not $R$-isomorphic to $N_{j} / N$ when $i \neq j$. Suppose we did have $N_{i} / N R$-isomorphic to $N_{j} / N$ when $i \neq j$ where we may assume $i \neq 1$. Choose an element $n$ in $N_{i}$ not in the center of $N_{i} / N$ and let $\tau$ denote the inner automorphism $n$ induces on $G$ (which lies in $R$ because $R$ is compatible). As the elements of $N_{i} / N$ and $N_{j} / N$ commute, $\tau$ acts as the identity on $N_{j} / N$. But then $\tau$ acts as the identity on $N_{i} / N$ as well which is impossible.

Because $R$ has dccr, $R$ has finitely many isomorphism classes of minimal modules. Hence our index set $I$ is finite so we might as well let $I=\{1,2, \ldots, n\}$. Since each $N_{i} / N$ is monogenic, let us write $N_{i} / N=\left(g_{i}+N\right) R$ where $g_{i} \in N_{i}$. Set

$$
g=\sum_{i=1}^{n} g_{i}
$$

We are going to show that

$$
G / N=(g+N) R
$$


This will follow if we can show each $g_{i}+N$ lies in $(g+N) R$. From the proof of [3, Theorem 10.41], for each $i>1$ there exists an element $\pi_{i} \in R$ such that $\pi_{i}$ acts as the identity on $N_{i} / N$ and $\pi_{i}$ annihilates $N_{j} / N$ for $j \neq i$. Thus for $i>1$,

$$
g_{i}+N=(g+N) \pi_{i} \in(g+N) R .
$$

Also if $i=1$,

$$
g_{1}+N=(g+N)\left(1-\sum_{i=2}^{n} \pi_{i}\right) \in(g+N) R
$$

and we have (1).

We complete the proof by showing $g R=G$. Were this not the case, some maximal $R$-ideal $M$ of $G$ would contain $g R$. But then

$$
(g R+N) / N \subseteq M / N \neq G / N
$$

violating (1).

We are now ready to prove our theorem. In our proof, the automorphism and inner automorphim groups of a group $G$ will be denoted $\operatorname{Aut}(G)$ and $\operatorname{Inn}(G)$, respectively. Also, the inner automorphism of $G$ induced by an element $g \in G$ will be denoted $\tau_{g}$.

Suppose that $\psi$ is a near-ring isomorphism from $R$ onto $S$. Since $G^{\prime}=G, G$ is a monogenic $R$-module by Lemma 4 . Hence the set $A$ of invertible distributive elements of $R$ are automorphisms of $G$ by Lemma 3. Thus $A=A u t(G) \cap R$ and $A$ additively generates $R$. The same holds for $A \psi$ relative to $H$ and $S$. Let us set

$$
K=\operatorname{Inn}(H) \psi^{-1} \text {. }
$$

$K$ is then a normal subgroup of $A$. Finally, let

$$
N=\operatorname{Inn}(G) \cap K
$$

and let $L$ be the normal subgroup of $G$ corresponding to $N$ under the natural homomorphism from $G$ onto $\operatorname{Inn}(G) . L$ is in fact an $R$-ideal of $G$, for suppose $l \in L$ and $\sigma \in A$. We have $\left(\tau_{l}\right)^{\sigma}=\tau_{l \sigma}$ lies in $N$. This then gives us that $l \sigma$ lies in $L$ and hence $L$ is an $R$-ideal of $G$ as claimed. Now we can view $G / L$ as being an $R$-module.

We are next going to see that $K$ acts trivially on $G / L$. Letting $g$ be an element of $G$ and $\sigma$ be an element of $K$, the commutator $\left[\tau_{g}, \sigma\right]$ is an element of $N$ and hence has the form $\tau_{l}$ for some $l \in L$. Thus

$$
\tau_{g \sigma}=\left(\tau_{g}\right)^{\sigma}=\tau_{g}\left[\tau_{g}, \sigma\right]=\tau_{g} \tau_{l}=\tau_{g+l} .
$$

Hence $g \sigma=g+l+z$ for some $z \in Z(G)$. Since $Z(G)$ is contained in $L$, we then have that $\sigma$ and hence $K$ act trivially on $G / L$.

Suppose that $L$ is properly contained in $G$. Let $M$ be a maximal $R$-ideal of $G$ containing $L$. As $G / M$ is a minimal $R$-module, it follows from [4, Theorem 1.2] that there are $S$-modules $P$ and $Q$ of $H$ with $P \subseteq Q$ and a group isomorphism $\varphi$ from $G / M$ onto $Q / P$ such that

$$
(x r) \varphi=(x \varphi)(r \psi)
$$

for each $x \in G / M$ and $r \in R$. As $K$ acts trivially on $G / M$, Inn $(H)$ acts trivially on $Q / P$. But then $Q / P$ is abelian and hence $G / M$ is abelian which is impossible since $G$ is perfect. Thus $L=G$. 
Because $L=G$, we now have that $\operatorname{Inn}(G)$ is contained in $K=\operatorname{Inn}(H) \psi^{-1}$. By symmetry we also have that $\operatorname{Inn}(H)$ is contained in $\operatorname{Inn}(G) \psi$. Thus $\psi$ is an isomorphism from $\operatorname{Inn}(G)$ onto $\operatorname{Inn}(H)$ and our theorem follows.

\section{REFERENCES}

[1] C. G. Lyons and G. Mason, Endomorphisms of near-rings of dicyclic groups and generalized dihedral groups, Proc. Royal Irish Acad., 91A(1991), 99-111. MR 93a:16038

[2] C. G. Lyons and J. D. P. Meldrum, $N$-series and tame near-rings, Proc. Royal Soc. Edinburgh, 86A(1980), 153-163. MR 82d:16033

[3] J. D. P. Meldrum, Near-Rings and their links with groups, Pitman, London, 1985. MR 88a:16068

[4] G. L. Peterson, On the structure of an endomorphism near-ring, Proc. Edinburgh Math. Soc., 32(1989), 223-229. MR 90e: 16063

[5] S. A. Syskin, On a problem of C. Lyons and G. Mason, to appear

[6] S. D. Scott, Tame near-rings and N-groups, Proc. Edinburgh Math. Soc., 23(1980), 275-296. MR 83b:16032

[7] S. D. Scott, On the finiteness and uniqueness of certain 2-tame $N$-groups, Proc. Edinburgh Math. Soc., 38(1995), 193-205. MR 96d:16058

Department of Mathematics, James Madison University, Harrisonburg, Virginia 22807

E-mail address: peterson@math.jmu.edu 